\title{
Obsessive-Compulsive Disorder During the COVID-19 Pandemic: a Systematic Review
}

\author{
Andrew G. Guzick ${ }^{1}$ (1) - Abigail Candelari ${ }^{1}$ - Andrew D. Wiese ${ }^{1} \cdot$ Sophie C. Schneider ${ }^{1} \cdot$ Wayne K. Goodman ${ }^{1}$. \\ Eric A. Storch ${ }^{1}$
}

Accepted: 19 August 2021 / Published online: 6 October 2021

(c) The Author(s), under exclusive licence to Springer Science+Business Media, LLC, part of Springer Nature 2021

\begin{abstract}
Purpose of Review This systematic review evaluated the impact of the COVID-19 pandemic on obsessive-compulsive symptoms.

Recent Findings Most studies showed that obsessive-compulsive symptoms worsened during the early stages of the pandemic, particularly for individuals with contamination-related obsessive-compulsive disorder (OCD), though other symptoms dimensions were found to worsen as well. Many patients and individuals in the general population experienced new obsessive-compulsive-like symptoms centered on COVID-19. Self-reported rates of symptom exacerbation and COVID19-focused symptoms were consistently lower in studies that recruited patients from specialty clinics (compared to online samples). Most studies were conducted in Spring/Summer, 2020.

Summary The COVID-19 pandemic has been an enormous stressor for individuals with OCD, especially for those with contamination symptoms. Regardless, there is strong reason to believe gold standard treatment approaches for OCD have maintained strong efficacy. Disseminating and effectively delivering evidence-based treatments for OCD is an urgent public health priority.
\end{abstract}

Keywords OCD · Contamination · Exposure and response prevention · Cognitive-behavioral therapy $\cdot$ Serotonin reuptake inhibitors

\section{Introduction}

As the COVID-19 pandemic has affected nearly every aspect of daily life across the globe, mental health professionals and communities at large raised alarm over the potential psychological impact of the pandemic. The psychological functioning of individuals with obsessive-compulsive disorder (OCD) has been a particular concern [1,2], as OCD is often characterized by recurrent fears of contamination and illness, active avoidance of potentially contaminated places and situations, and excessive washing [3]. It is conceivable, then, that public health mandates calling for intensified

This article is part of the Topical collection on Anxiety Disorders

Andrew G. Guzick

Andrew.guzick@bcm.edu

1 Department of Psychiatry and Behavioral Sciences, Baylor College of Medicine, 1977 Butler Blvd., Suite 4-100

Houston, TX 77030, USA protective measures like physical distancing and frequent handwashing, as well as widespread attention directed toward the potential threat of the virus, were enormous stressors for individuals with OCD.

Clinical researchers across the globe began investigating OCD during the pandemic and wondered whether OCD concerns would begin centering on COVID-19. This was particularly the case during early stages of the pandemic, when public alarm about the virus was at its height and mandated lockdowns were widespread [1,4]. Similarly, investigators and clinicians alike were interested in understanding the course of obsessive-compulsive symptoms during the pandemic, and whether certain factors (e.g., obsessions and compulsions related to contamination) would be associated with a poorer prognosis $[5 \bullet, 6]$. This literature has become quite developed over the course of the pandemic and may allow conclusions to be drawn regarding the impact of the pandemic on obsessive-compulsive symptoms, both among patients with OCD and the general population. Reviewing this literature may inform evidence-based approaches to 
support this population as the pandemic continues to impact daily life across the globe, as well as how to prepare for the mental health impact of future pandemics or other largescale crises.

The central questions that guided this review are as follows:

1. What has the course of obsessive-compulsive symptoms been during the pandemic, both in people with OCD and in the general population?

2. Has there been a particularly worse course for individuals with contamination-related OCD?

3. What has been the incidence and impact of COVID19-centered obsessive-compulsive symptoms during the pandemic?

4. What factors have been associated with obsessivecompulsive distress during the pandemic?

\section{Methods}

\section{Search Strategy}

On April 29, 2021, a literature search was conducted searching for articles assessing OCD during the COVID-19 pandemic. A PubMed search was conducted with the following terms: "OCD[Title/Abstract OR obsessive-compulsive [Title/Abstract]) AND (COVID-19[Title/Abstract] OR SARS-CoV-2[Title/Abstract]," and an EmBase search was also conducted with corresponding terms: ("obsessive compulsive disorder":ti,ab,kw OR “ocd":ti,ab,kw) AND (“covid19":ti,ab,kw OR “sars-cov-2":ti,ab,kw). Google Scholar alerts for studies of COVID-19 and OCD were also monitored until June 18, 2021. We also included a relevant study currently under review conducted by the author team. Results from these sources were combined after removing duplicates. Inclusion criteria were (1) the study investigated OCD or obsessive-compulsive symptoms during the pandemic, (2) the paper included an empirical study with data collection, and (3) the study was published in English. Case studies/ series and clinical commentaries or reviews were excluded. The final number of included studies for this review was 67. Please see Fig. 1 for a PRIMSA diagram of the search approach.

Findings from each study were extracted according to the following themes: (1) course of obsessive-compulsive symptoms during the pandemic, (2) impact of contamination symptoms, (3) COVID-19-centered symptoms, (4) factors associated with obsessive-compulsive symptoms during the pandemic. For each study, the nature of the sample (e.g., clinical vs. non-clinical), sample size, study location, general study design characteristics, and dates of data collection were also extracted. Please see Supplemental Table 1 for a summary of this information.

\section{Results}

\section{Course of Obsessive-Compulsive Symptoms during the Pandemic}

\section{Clinical Studies}

Several studies assessed OCD severity at multiple timepoints, both in clinical and non-clinical samples.

Longitudinal studies with clinical samples generally recruited patients with OCD being seen in specialty care settings and compared Yale-Brown Obsessive-Compulsive Scale (Y-BOCS) $[7,8]$ scores or other validated assessments from before the pandemic with their symptoms during the early stages of the pandemic. Three studies with adults and one with youth found significant increases in OCD severity during the early stages of the pandemic compared with prior to the pandemic using validated symptom assessments

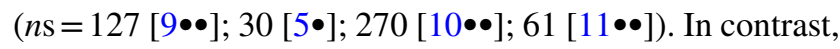
one study found improvement from treatment initiation to just prior to the pandemic to just after the pandemic began and found no difference in OCD symptom course compared with another cohort followed at their clinic a year earlier $(n=240)[12 \bullet \bullet]$. Another study found a lack of change in OCD symptoms among youth, though this was conducted with a smaller sample $(n=29)$ and used a single-item rating rather than a gold-standard assessment [13].

A more common approach in evaluating symptom course during the pandemic was through asking individuals with OCD whether their symptoms worsened during the pandemic. Studies recruited patients actively involved in treatment in specialty clinical settings or online/through prior participation in OCD research. In the clinical studies, estimates of worsened symptoms were $6 \%(n=84)$ [14], $10 \%(n=60)$ [6], 12\% $(n=113)$ [15], 32\% $(n=65)$ [16], $36 \%(n=123)[17 \bullet], 45 \%(n=65)[18]$, and $65 \%(n=127)$ $[9 \bullet \bullet$. The pooled rate was calculated to be $32 \%$ (95\% CIs $28-35 \%$ ). Notably, two of the three studies with the highest estimates of worsened symptoms were from studies conducted in Italy during the early stages of the pandemic, which was among the most severely impacted nations [ $[9 \bullet \bullet$, $17 \bullet]$. One study with youth with OCD $(n=29)$ found that significantly more were rated as "improved" on the Clinical Global Impressions (CGI)-Improvement Scale (45\%) than those who were "worsened" (10\%), with no significant change in CGI-Severity Scale scores across assessments [11••]. Experienced exposure and response prevention clinicians provided an estimate near the middle of this range, reporting that approximately $38 \%$ on average worsened 


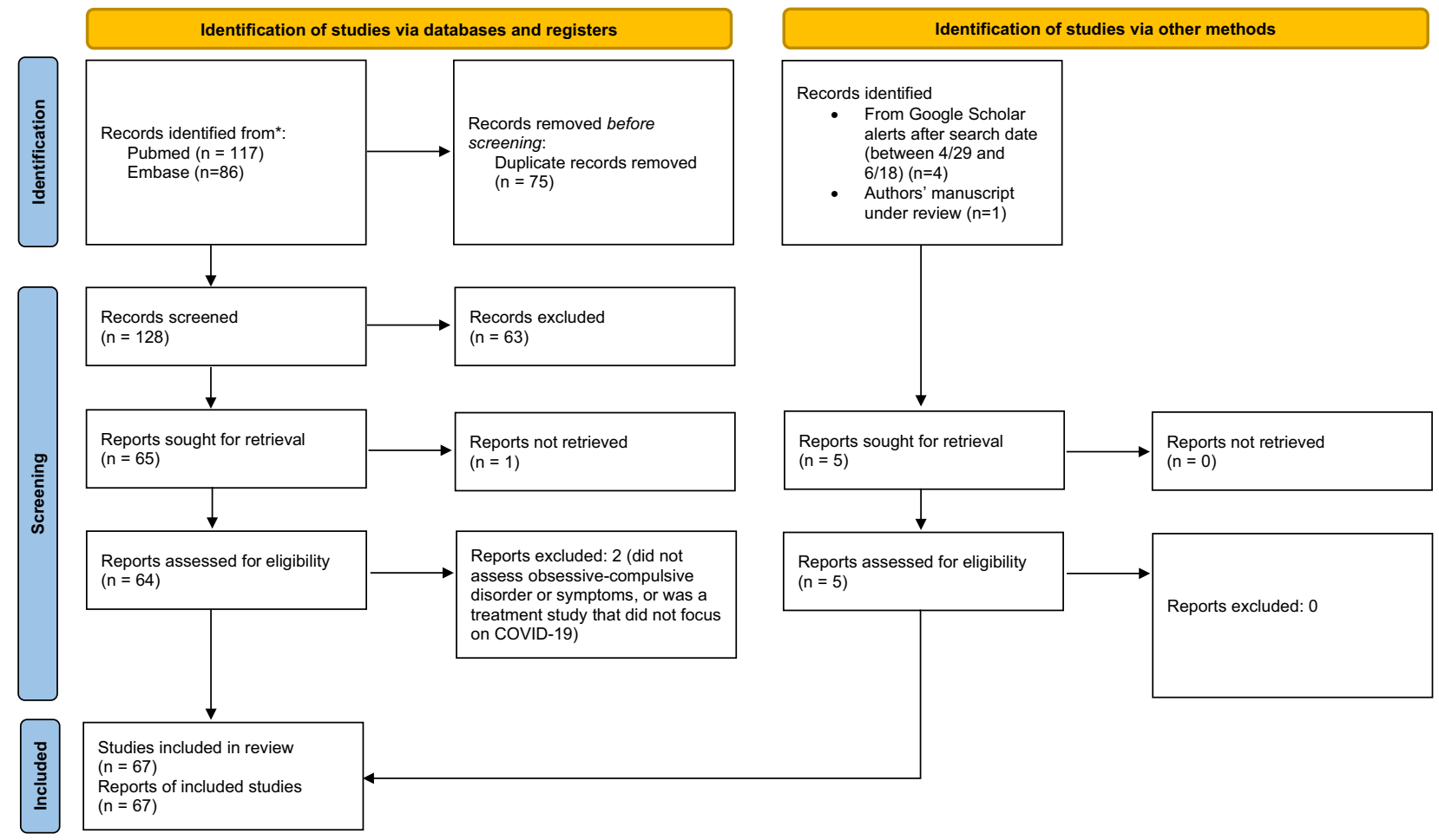

From: Page MJ, McKenzie JE, Bossuyt PM, Boutron I, Hoffmann TC, Mulrow CD, et al. The PRISMA 2020 statement: an updated guideline for reporting systematic reviews. BMJ 2021;372:n71. doi: 10.1136/bmj.n71. For more information, visit: http://www.prisma-statement.org/

Fig. 1 PRISMA 2020 flow diagram: OCD and COVID-19. From: Page MJ, McKenzie JE, Bossuyt PM, Boutron I, Hoffmann TC, Mulrow CD, et al. The PRISMA 2020 statement: an updated guideline for reporting

during the pandemic ( $n=137$ clinicians) [19]. Rates of worsening were higher in the online samples, including $36 \%(n=47)$ [20], 73\% $(n=37)$ [18], 76\% $(n=394)$ [21 •], $76 \%(n=252)$ [22], 93\% $(n=196)$ [23], resulting in a pooled estimate of $77 \%$ (95\% CIs 75-80\%).

\section{Non-clinical Studies}

A small number of studies also assessed obsessive-compulsive symptoms prospectively across multiple timepoints during the pandemic in non-OCD samples, yielding less consistent findings. In one large university sample in China $(n=5,827)$, estimates of elevated OCD based on self-report Y-BOCS scores in February, March, and April, 2020 were 11.3\%, 3.6\%, and 3.5\%, respectively [24•]. Another study with college students also found OCD symptoms decreased from before the pandemic, to mid-lockdown, to after the lockdown in Italy [25]. In contrast, Loosen and colleagues [26•] assessed obsessive-compulsive symptoms in 406 adults in the UK in April/May, 2020 and again in July/August, 2021, finding significantly increased obsessivecompulsive symptoms. Similarly, OCD symptoms were also observed to increase from pre-pandemic to the early stages of the pandemic in youth with neuropsychiatric disorders [27]. A final systematic reviews. BMJ 2021;372:n71. https://doi.org/10.1136/bmj. n71. For more information, visit: http://www.prisma-statement.org/

study found that adults with ASD (without OCD) were not found to have significantly greater obsessive-compulsive symptoms from before to after the lockdown using a brief screener [28].

More broadly, several studies found higher-than-expected rates of clinically elevated OCD in the general population using established cut-point on the Obsessive-Compulsive Inventory-Revised, including 12\% in Portugal in March (29) and 21\% in Germany in March/April [30] or when comparing symptoms with norm values in China in February (31) as well as in the UK in April/May and July/August [26•].

\section{Impact of Contamination on Symptom Severity, Course, and Other Outcomes}

\section{Clinical Studies}

A number of studies that assessed individuals with OCD evaluated the impact of contamination symptoms on symptom severity and course.

Six studies found evidence for a poorer course for those with contamination symptoms, including three that found having contamination-related OCD prior to the pandemic predicted greater increases in overall symptom severity 
during the pandemic $(n \mathrm{~s}=30[5 \bullet], 127[9 \bullet \bullet]$, and $394[21 \bullet])$. Importantly, this was the case in two longitudinal studies investigating this question $[5 \bullet, 9 \bullet \bullet$. Similarly, a longitudinal study with youth found significantly higher rates of contamination symptoms but not other dimensions during the pandemic [11••]. In their prospective study of patients with OCD $(n=270)$, Khosravani and colleagues found that OCD symptoms worsened in all domains, including contamination as well as symmetry/completeness, harm, and unacceptable thoughts $[10 \bullet \bullet]$. Although all domains significantly worsened, effect sizes appeared largest for contamination and comparisons of whether increases were greater for the contamination group were not conducted. A retrospective evaluation $(n=196)$ found higher worsening in those with contamination compared with symmetry symptoms (though no differences with harm or unacceptable thoughts were noted) [23]. A study of patients with remitted or partially remitted OCD found that all patients with worsened OCD during the pandemic (based on repeated Y-BOCS administrations) had a primary contamination presentation [6].

In contrast, five studies did not find evidence for differences in course among those with contamination symptoms. This included one longitudinal study $(n=240)$ [12••], three retrospective studies $(n s=113$ [15], 123 [16], and 65 [17•), and in one asking for clinician retrospective reports of their patients' symptoms during the course of the pandemic [19].

Together, although these findings were overall mixed, it should be noted that three longitudinal studies that directly compared the course of individuals with contamination vs. other symptoms found a poorer course for contamination $[5 \bullet, 9 \bullet \bullet, 11 \bullet \bullet]$, compared with one that did not find a difference (though this study was an outlier in terms of minimal overall worsening in patients with OCD) [12••]. Two studies specifically compared symptom domain scores across time, finding significantly worsened contamination, harm, and taboo-related symptoms during the pandemic [10••, 23], though direct comparisons of trajectories across symptom domains were not conducted.

Contamination symptoms in individuals with OCD were associated with increased COVID-19-related stress [32], COVID-related fear [22], new OCD symptoms focused on COVID-19 [9••], and COVID-19 dysfunctional beliefs (e.g., that the pandemic was caused by people being careless with hygiene, that fears/dangers about the world are confirmed) $[21 \bullet]$.

\section{Non-clinical Studies}

Among 6041 individuals texting a support line in March, 2020, 60\% endorsed obsession-like thoughts related to contamination and excessive washing, which were associated with increased distress levels $[33,34]$. Contamination symptoms were found to increase during the pandemic in college students [35], non-clinical adults [26•, 36]. One of these studies found that contamination-related OCD symptoms were similar to previously validated clinical samples and significantly greater than non-clinical samples across timepoints (April/May and again in July/August) [26•]. Finally, in one study of patients with ST-elevation myocardial infarction, contamination-related OCD symptoms were associated with significant delays in going to the hospital [37].

\section{COVID-19-Centered Obsessive-Compulsive Symptoms}

\section{Clinical Studies}

Studies investigated whether people began experiencing obsessions and compulsions with COVID-19 themes. A divide in frequency across samples recruited from clinic vs. online settings was again observed. In studies with online participants who self-identified with OCD, estimates of new, COVID-centered obsessions and compulsions were $35 \%$ in a youth sample $(n=37[18])$ and $58 \%$ in an adult sample $(n=252$ [22]), for a pooled estimate of $55 \%$ (95\% CI 47-58\%). In contrast, rates of new COVID19-centered obsessions and compulsions were generally lower in samples recruited from patients being treated in specialty clinics, including $4 \%(n=113)$ [15], 5\% $(n=240)$ [12••], and 15\% $(n=65)$ [18]. A study conducted in Italy was an exception to this pattern $(n=127)$, with $45 \%$ experiencing COVID-19-focused obsessions and compulsions early in the pandemic $[9 \bullet \bullet]$. Across these four studies, the pooled incidence of new COVID-19-focused symptoms among patients being followed in clinic was 16\% $(95 \%$ CI 13-20\%).

Relatedly, COVID-19-stress syndrome has now been a widely studied construct, a cluster of stress responses to the pandemic including socio-economic consequences, traumatic stress, xenophobia, and most notably, danger/ contamination fears and compulsive checking, which could be conceptualized as COVID-19-centered COVID19 symptoms [38]. Unsurprisingly, it appears that greater OCD severity, especially contamination and checking symptoms, are associated with all these COVID-19 stress symptoms, including contamination fears and excessive COVID-19-related checking [32]. This study found that patients with OCD had greater stress across COVID-19 stress syndrome domains than patients with social anxiety disorder or specific phobias, and similar levels as those with panic and generalized anxiety disorders [32]. 


\section{Non-clinical Studies}

Non-clinical studies also showed the increased obsessivecompulsive symptom severity was associated with more intrusive thoughts and repetitive behaviors centered on COVID-19 [39,40], as well as COVID-19-related safety behaviors [41]. Contamination symptoms specifically were also associated with COVID-19 safety behaviors [41]. One of these studies adapted a standard OCD measure to assess COVID-specific concerns found that certain repetitive thoughts and behaviors were relatively prevalent in the general Chinese population, with $18 \%$ endorsing repetitively telling others to take precautions and $12 \%$ endorsing excessive news-checking.

\section{Factors Associated With Obsessive-Compulsive Symptoms During the Pandemic}

\section{Clinical Studies}

People with OCD have been found to have more pandemic-

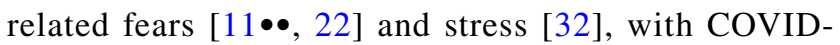
19-related stress predicting a poorer longitudinal course of obsessive-compulsive symptoms in one study [10••]. Another study found that financial stress caused by the pandemic, pathological doubt, and being an adult (vs. a child), and being medically at risk for COVID-19 all corresponded with a poorer course of exposure and response prevention treatment [19]. Greater pre-pandemic depression, OCD severity, and less social support all predicted greater exacerbations of OCD severity as well $[9 \bullet \bullet]$. In one of the only studies of youth with OCD during the pandemic, Tanir and colleagues found that attention toward COVID-19-related information, OCD duration, and knowing someone who had COVID-19 all predicted OCD exacerbations [11 • ].

Studies that compared individuals with OCD with healthy controls found that individuals with OCD experienced more internalizing symptoms, suicidal thoughts, sleep and appe-

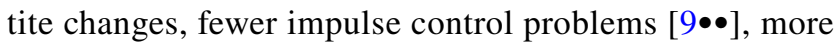
COVID-related stress [20], and fear [22], as well as poorer coping strategies and less social support [42]. One study compared mental health trajectories among individuals with psychiatric diagnoses, including OCD, with healthy controls [43]. This study found greater increases in mental health symptoms in individuals without psychiatric diagnoses relative to people with more comorbid diagnoses (including OCD, anxiety disorders, and depressive disorders), though it is important to note individuals with OCD specifically were not evaluated nor were obsessive-compulsive symptoms as an outcome.

OCD severity during the pandemic has also been found to be directly correlated with suicidal ideation $[9 \bullet \bullet, 17 \bullet, 44]$, poor coping [42,45-49], sleep disturbances [17•], family accommodation [17•], family conflict [46], avoidance [18], anxiety [18], depression [18], and medication adjustments $[9 \bullet \bullet, 17 \bullet]$. A greater number of OCD consults were seen in one emergency room psychiatry consult service during March, 2020, compared with March, 2019 [50].

\section{Non-clinical Studies}

In non-clinical samples, multiple studies showed that increased obsessive-compulsive symptoms were associated with increased adherence to guidelines [26•, 51], underscoring the potential adaptive role of obsessive-compulsive symptoms (e.g., washing, checking) in the presence of a true viral threat. In one of these studies, information-seeking in April/May, 2020 predicted increased OCD symptoms in July/August, 2020, which in turn predicted greater guideline adherence [26•]. That said, in a representative USA sample, COVID prevention behaviors were associated with having clinically elevated OCD symptoms, both broadly assessed and specific to contamination [41]. Relatedly, COVID-19 media consumption was also associated with OCD in college student sample [52].

COVID-19-related fears were also associated with OCD severity in non-clinical samples [53,54]. Other factors associated with OCD included intolerance of uncertainty [53], being at risk for COVID-19 [41], emotional contagion [52], family conflict [55], loss of income [56], younger age [29,56], female gender [56,57], less psychological resilience [30], and psychiatric comorbidity [58].

A number of studies investigated whether healthcare workers experienced more obsessive-compulsive symptoms. Associations were found between healthcare worker status and compulsions [59], contamination symptoms [60], and overall OCD severity $[57,61,62]$, though one did not find a difference in OCD severity [63].

\section{Discussion}

As anticipated [1,2], COVID-19 has proven to be an enormous stressor for individuals with OCD. A wide body of literature now shows that that obsessive-compulsive symptoms increased during the early stages of the pandemic, both in those with OCD and the general population. COVID-19 became a central theme for many people with OCD, particularly those with contamination symptoms independent of COVID-19, more COVID-related stress, and more fear of COVID-19. Although multiple studies found that obsessivecompulsive symptoms worsened across symptom dimensions, high-quality studies (those that used longitudinal designs, use of repeated validated assessments) that directly compared outcomes across domains found evidence for a poorer course among patients with contamination 
symptoms in three of four studies. When comparing studies conducted within specialty clinics vs. those that recruited online samples, participants in specialty care reported less impact from the pandemic, underscoring the importance of evidence-based treatment to mitigate the detrimental impact of the pandemic for many with OCD. OCD has been associated with several psychosocial factors during the pandemic, with poor coping, suicidal ideation, and situational COVID19-related stressors (e.g., losing income, knowing someone who was sick, or working in healthcare) being the most replicated variables in addition to COVID-19-related fear, further highlighting the need to extend outreach to individuals with OCD who have suffered most during the pandemic.

This pattern of findings speaks to our earlier calls for continued robust delivery of evidence-based treatments for OCD, including exposure and response prevention and serotonin reuptake inhibitor therapy [2,64]. Although $32 \%$ of patients with OCD in specialty clinics self-reported worsening symptoms during the pandemic across studies, as well as $16 \%$ with new COVID-19-related symptoms, in samples recruited outside specialty care, these estimates were $77 \%$ and $55 \%$, respectively. Thus, being under the care of experienced OCD specialists appears to mitigate the harms of the pandemic, and in many cases likely results in clinical improvement.

During non-pandemic times, most therapists have been hesitant to deliver exposure and response prevention with the intensity that has been shown to produce best outcomes (e.g., minimizing avoidance and safety behaviors, engaging in exposures that are highly anxiety provoking and thus effectively target obsessive fears) [65]. It is conceivable that many therapists will deliver this treatment especially cautiously for individuals with contamination-related symptoms even after vaccines have been widely adopted and the threat of the virus is lower, as patterns of active avoidance that developed during this time may persist. Considering the immense suffering many people with contamination-based OCD experienced during the pandemic, it is imperative that clinicians follow public health guidelines without overestimating the threat of the virus themselves $[2,66]$. With the rapid adoption of telehealth that has occurred in the wake of the pandemic, psychological and psychiatric treatment remains feasible for many people with OCD. It will be important to continue monitoring both patients with OCD and clinicians when the world begins to slowly recover from the pandemic, but residual apprehension and new norms around contamination, cleaning, and social distancing may remain in place.

Although trajectories and rates of clinical worsening were relatively better in clinical samples, it is important to note there was still a larger than expected rate of clinical worsening during the pandemic among patients under specialty care, with symptoms significantly increasing in five of six longitudinal studies. Despite these follow-up periods spanning multiple months, which generally corresponds with at least part of a course of evidence-based psychological or pharmacological therapy, many of these studies found high rates of no change or worsening. Thus, although specialty care may have minimized the impact of the pandemic for many, treatment outcomes may have been less robust than what clinicians aim for in typical times.

When viewing the pattern of results from the studies in this review, there do appear to be a range of outcomes, with some finding minimal impact from the pandemic and others finding a substantial detriment. These at times contrasting results likely reflect different states of the pandemic in different parts of the world at different snapshots in time. Two examples demonstrate the importance of considering location and timing when interpreting the literature that has emerged during this crisis. One of these examples comes from examining two studies from Italy early in the pandemic, one of the most impacted countries during the early stages. As one might expect, these studies showed higher rates of clinical worsening than other studies using similar methods $[9 \bullet \bullet, 17 \bullet]$. A longitudinal study from China highlights the importance of considering timing and geography; in this study, investigators followed thousands of university students in February, March, and April; self-reported OCD severity above a clinical cutoff was $11 \%$ in February, but had already dropped to typical levels by March and April [24•]. This study suggests OCD exacerbations may have reflected a relatively short-lived acute stress response in the general population in China, though studies conducted in the UK, the USA, and Iran found that obsessive-compulsive symptoms either maintained or worsened through mid-tolate summer in clinical and non-clinical samples $[10 \bullet \bullet, 19$, 26•]. When viewing findings together, the data are clear that the pandemic has placed significant strain on the wellbeing of those with OCD, though the degree of impact is highly dependent on the particular circumstances of the pandemic at a given point in time and geographical are.

Although there was evidence for a particularly worse course for those with contamination symptoms, several studies found that obsessive-compulsive symptoms increased across domains. This suggests that COVID-19 has been both a broad stressor that may lead to increased obsessive-compulsive symptoms regardless of symptom content, though has also had a specific effect on those with contamination symptoms in particular, as contamination symptoms were also associated with COVID-19-related distress more specifically in several studies.

A few studies found that public health guideline adherence was cross-sectionally and longitudinally associated with obsessive-compulsive severity $[26 \bullet, 51]$ (though see one exception [15]). This may reflect what has been called the "behavioral immune system," or an adaptive change in 
behaviors (e.g., checking, washing) in response to a true viral threat [66]. Clearly, however, this pattern can become maladaptive when it is excessive and irrational (for example, individuals who continued to clean their groceries after it was determined there was a low risk of COVID-19 spreading via surfaces). It would be interesting to know whether this change in behavior has translated to fewer infections in individuals with OCD, though to our knowledge, this question has not been directly addressed. Carefully assessing pathological vs. normative safety behaviors in the context of a pandemic will continue to be an important element of psychiatric assessment.

Although, beyond the scope of this review, it is worth noting there is strong preliminary evidence drawing an association between SARS-CoV-2 infection and OCD as well $[67,68]$, and there are well-documented mood and anxiety sequelae related to COVID-19 [69]. It is likely that both the psychological stress the virus causes and the central nervous system inflammation caused by the immune response itself contribute to increased risk for OCD in this population $[67,68]$. Indeed, elevated obsessive-compulsive symptoms were found in $20 \%$ of 402 COVID-19 survivors in one report at 1-month follow-up [67], which were found to worsen 2 months later in a subsample of this cohort [68]. Research on the neuropsychiatric sequelae of SARS-CoV-2 should continue to include obsessive-compulsive symptoms as an important outcome.

A notable limitation of this review was that a vast majority of research was conducted in the first months of the pandemic, and it is much less clear how the longer course has been for patients with OCD. That said, the few studies with follow-ups into late summer or fall, 2020, generally did not show an improved course for those with OCD [10••, $19,22,23,26 \bullet]$. Continued monitoring of the literature is highlighted in order to observe whether further follow-ups are conducted, which may offer continued guidance for how clinicians may best support this population. Another limitation of this review was the difficulty in accurately aggregating results across studies that frequently used study-specific assessments that were not psychometrically validated or were retrospectively rated. That said, in drawing conclusions for this paper, we focused on summarizing replicated findings from studies with stronger designs.

\section{Conclusions}

The COVID-19 pandemic has proven to be a tremendous stressor for both the general population and individuals with OCD, with many experiencing exacerbations in obsessivecompulsive symptoms and the emergence of new symptoms focused on COVID-19. Regardless, there is good reason to believe that evidence-based treatments maintained efficacy during this time and mitigated the impact of the pandemic for many people. Dissemination and effective delivery of evidence-based therapies for children and adults with OCD remains a top priority for our field, a priority that has become even more urgent during the COVID-19 pandemic.

Supplementary Information The online version contains supplementary material available at https://doi.org/10.1007/s11920-021-01284-2.

Funding Research reported in this publication was supported by the Eunice Kennedy Shriver National Institute of Child Health \& Human Development of the National Institutes of Health under Award Number P50HD103555 for use of the Clinical and Translational Core facilities.

\section{Compliance with Ethical Standards}

Disclaimer The content is solely the responsibility of the authors and does not necessarily represent the official views of the National Institutes of Health.

\section{References}

Papers of particular interest, published recently, have been highlighted as:

- Of importance

$\bullet$ Of major importance

1. Banerjee DD. The other side of COVID-19: impact on obsessive compulsive disorder (OCD) and hoarding. Psychiatry Res. 2020;288:112966.

2. Storch EA, Schneider SC, Guzick A, McKay D, Goodman WK. Impact of COVID-19 on exposure and response prevention for obsessive-compulsive disorder: present and post-pandemic considerations. Psychiatry Res. 2020;292:113310.

3. Bloch MH,Landeros-Weisenberger A, Rosario MC, Pittenger C, Leckman JF. Meta-analysis ofthe symptom structure of obsessivecompulsive disorder. Am J Psychiatry. 2008;165(12):1532-42.

4. French I, Lyne J.Acute exacerbation of OCD symptoms precipitated by media reports of COVID-19.Ir J Psychol Med. 2020;37(4):291-4.

5. Davide P. The impact of the COVID-19 pandemic on patients with OCD: effects of contamination symptoms and remission state before the quarantine in a preliminary naturalistic study. Psychiatry Res. 2020;291:113213. First longitudinal report of OCD during the pandemic, finding symptom worsening that was associated with contamination symptoms.

6. Matsunaga H, Mukai K, Yamanishi K. Acute impact of COVID19 pandemic on phenomenological features infully or partially remitted patients with obsessive-compulsive disorder.Psychiatry Clin Neurosci. 2020;74(10):565-6.

7. Goodman WK, Price LH, Rasmussen SA, Mazure C, Fleischmann RL, Hill CL, et al. The Yale-Brown Obsessive Compulsive Scale: I. Development, Use, and Reliability. Arch Gen Psychiatry. 1989;46(11):1006-11.

8. Goodman WK. TheYale-Brown Obsessive Compulsive Scale: II. Validity. Arch Gen Psychiatry. 1989;46(11):1012.

9.• Alonso P, Bertolín S, Segalàs J, Tubío-Fungueiriño M, Real E, Mar-Barrutia L, et al. How is COVID-19 affecting patients with obsessive-compulsive disorder? A longitudinal study on the initial phase of the pandemic in a Spanish cohort. Eur Psychiatry 
[Internet]. 2021;64(1). Available from: https://www.cambridge. org/core/journals/european-psychiatry/article/how-is-covid19affecting-patients-with-obsessivecompulsive-disorder-a-longitudinalstudy-on-the-initial-phase-of-the-pandemic-in-a-spanish-cohort/ 79711647FF8B8D96A2F8E93FC96E4C06. Large longitudinal study of the impact of COVID on OCD $(n=127)$, finding clinically significant symptom worsening in $40 \%$ of patients, particularly those with contamination symptoms. Found more problems with suicidal ideation in those with $\mathrm{OCD}$ relative to controls.

10.• Khosravani V, Aardema F, Samimi Ardestani SM, Sharifi Bastan F. The impact of the coronavirus pandemic on specific symptom dimensions and severity in OCD: a comparison before and during COVID-19 in the context of stress responses. J ObsessiveCompuls Relat Disord. 2021;29:100626. This large study of patients with $\mathrm{OCD}(\mathrm{n}=\mathbf{2 7 0})$ documented a significant relationship between worsened OCD symptoms across domains and COVID-19 stress responses. They documented significant increases in OCD severity across contamination, harm, taboo thought, and symmetry dimensions.

11.• Tanir Y, Karayagmurlu A, Kaya İ, Türkmen G, Dambasan BN. Exacerbation of obsessive compulsive disorder symptoms in children and adolescents during COVID-19 pandemic. Psychiatry Res. $2020 ; 293: 113363$. This was the only longitudinal study in youth with OCD that used validated, multi-item assessments, finding exacerbated symptoms in youth during the first months of the pandemic.

12.• Sharma LP, Balachander S, Thamby A, Bhattacharya M, Kishore $\mathrm{C}$, Shanbhag V, et al. Impact of the COVID-19 pandemic on the short-term course of obsessive-compulsive disorder. J Nerv Ment Dis. $2021 ; 209(4): 256-64$. This study compared the course of patients with OCD during the pandemic with patients across the same timeframe before the pandemic, finding minimal impact from the pandemic. This study was an outlier among the longitudinal studies with clinical populations, finding minimal rates of symptom worsening during the pandemic.

13. Schwartz-Lifshitz M, Basel D, Lang C, Hertz-Palmor N, Dekel I, Zohar J, et al. Obsessive compulsive symptoms severity among children and adolescents during COVID-19 first wave in Israel. J Obsessive-Compuls Relat Disord. 2021;28:100610.

14. Chakraborty A,Karmakar S. Impact of COVID-19 on obsessive compulsive disorder (OCD). Iran JPsychiatry. 2020;15(3):256-9.

15. Carmi L, Ben-Arush O, Fostick L, Cohen H, Zohar J. Obsessive compulsive disorder during COVID-19-two- and six-month followups. OCD during COVID-19. Int J Neuropsychopharmacol[Internet]. 2021. https://doi.org/10.1093/ijnp/pyab024.

16. Littman R,Naftalovich H, Huppert JD, Kalanthroff E. Impact of COVID-19 onobsessive-compulsive disorder patients. Psychiatry Clin Neurosci. 2020;74(12):660-1.

17. Benatti B, Albert U, Maina G, Fiorillo A, Celebre L, Girone $\mathrm{N}$, et al. What happened to patients with obsessive compulsive disorder during the COVID-19 pandemic? A multicentre report from tertiary clinics in Northern Italy. Front Psychiatry. 2020;11:720. Longitudinal study with 123 patients that documented high rates of symptom worsening, particularly among those with newly developed obsessions, more information-seeking on the Internet, and suicidal ideation.

18. Nissen JB, HøjgaardDRMA, Thomsen PH. The immediate effect of COVID-19 pandemic on children andadolescents with obsessive compulsive disorder. BMC Psychiatry. 2020;20(1):511.

19. Storch EA, Sheu JC, Guzick AG, Schneider SC, Cepeda SL, Rombado BR, et al. Impact of the COVID-19 pandemic on exposure and response prevention outcomes in adults and youth with obsessive-compulsive disorder. Psychiatry Res. 2021;295:113597.
20. Quittkat HL, Düsing R, Holtmann F-J, Buhlmann U, Svaldi J, Vocks S. Perceived impact of Covid-19 across different mental disorders: a study on disorder-specific symptoms, psychosocial stress and behavior. Front Psychol. 2020;11:586246.

21. Jelinek L, Moritz S, Miegel F, Voderholzer U. Obsessivecompulsive disorder during COVID-19: turning a problem into an opportunity? J Anxiety Disord. 2021;77:102329. This was a large study of individuals who were diagnosed with OCD in a prior research study, with $72 \%$ reporting symptom worsening during the pandemic. They documented greater rates of symptom worsening among those with contamination symptoms.

22. Wheaton MG, Ward HE, Silber A, McIngvale E, Björgvinsson T. How is the COVID-19 pandemic affecting individuals with obsessive-compulsive disorder (OCD) symptoms? J Anxiety Disord. 2021;102410.

23. Kaveladze B, Chang K, Siev J, Schueller SM. Impact of the COVID-19 pandemic on online obsessive-compulsive disorder support community members: survey study. JMIR Ment Health. 2021;8(2):e26715

24.• Ji G, Wei W, Yue K-C, Li H, Shi L-J, Ma J-D, et al. Effects of the COVID-19 pandemic on obsessive-compulsive symptoms among university students: prospective cohort survey study. J Med Internet Res. 2020;22(9):e21915. This large study of college students in China found clinically elevated OCD symptoms in $11 \%$ of participants early in the pandemic, which fell to typical rates later on.

25. Meda N, Pardini S,Slongo I, Bodini L, Zordan MA, Rigobello P, et al. Students' mental healthproblems before, during, and after COVID-19 lockdown in Italy. J Psychiatr Res.2021;134:69-77.

26. Loosen AM, Skvortsova V, Hauser TU. Obsessive-compulsive symptoms and information seeking during the Covid-19 pandemic. Transl Psychiatry. 2021;21(11):1-10. A large study of community adults found scores on an OCD assessment were similar to clinical levels across multiple months of follow-up during the pandemic in the United Kingdom.

27. Conti E, Sgandurra G, De Nicola G, Biagioni T, Boldrini S, Bonaventura E, et al. Behavioural and emotional changes during COVID-19 lockdown in an Italian paediatric population with neurologic and psychiatric disorders. Brain Sci. 2020;10(12).

28. Lugo-Marín J, Gisbert-Gustemps L, Setien-Ramos I, EspañolMartín G, Ibañez-Jimenez P, Forner-Puntonet M, et al. COVID19 pandemic effects in people with autism spectrum disorder and their caregivers: evaluation of social distancing and lockdown impact on mental health and general status. Res Autism Spectr Disord. 2021;83:101757.

29. Silva Moreira P, Ferreira S, Couto B, Machado-Sousa M, Fernández M, Raposo-Lima C, et al. Protective elements of mental health status during the COVID-19 outbreak in the Portuguese population. Int J Environ Res Public Health. 2021;18(4).

30. Munk AJL, Schmidt NM, Alexander N, Henkel K, Hennig J. Covid-19-beyond virology: potentials for maintaining mental health during lockdown. PloS One. 2020;15(8):e0236688.

31. Jiang R. Knowledge, attitudes and mental health of university students during the COVID-19 pandemic in China. Child Youth Serv Rev. 2020;119:105494.

32. Khosravani V, Asmundson GJG, Taylor S, Sharifi Bastan F, Samimi Ardestani SM. The Persian COVID stress scales (Persian-CSS) and COVID-19-related stress reactions in patients with obsessive-compulsive and anxiety disorders. J Obsessive-Compuls Relat Disord. 2021;28:100615.

33. Abba-Aji A, Li D, Hrabok M, Shalaby R, Gusnowski A, Vuong $\mathrm{W}$, et al. COVID-19 pandemic and mental health: prevalence and correlates of new-onset obsessive-compulsive symptoms in a Canadian province. Int $\mathbf{J}$ Environ Res Public Health. 2020;17(19). 
34. Robillard R, Saad M, Edwards J, Solomonova E, Pennestri $\mathrm{M}-\mathrm{H}$, Daros A, et al. Social, financial and psychological stress during an emerging pandemic: observations from a population survey in the acute phase of COVID-19. BMJ Open. 2020;10(12): 043805 .

35. Knowles KA, Olatunji BO. Anxiety and safety behavior usage during the COVID-19 pandemic: the prospective role of contamination fear. J Anxiety Disord. 2021;77:102323.

36. Cox RC, Olatunji BO. Linking insomnia and OCD symptoms during the coronavirus pandemic: Examination of prospective associations. J Anxiety Disord. 2021;77:102341.

37. Karagöz A, Keskin B,Kültürsay B, Ceneli D, Akbal OY, Tokgoz $\mathrm{HC}$, et al. Temporal association of contamination obsession on the prehospital delay of STEMI during COVID-19pandemic. Am J Emerg Med. 2021;43:134-41.

38. Taylor S. COVID stresssyndrome: clinical and nosological considerations. Curr Psychiatry Rep. 2021;23(4):19.

39. Acenowr CP, Coles ME. OCD during COVID-19: understanding clinical and non-clinical anxiety in the community. Psychiatry Res. 2021;300:113910.

40. Leong A, Colah Z, Guzick AG, Schneider SC, Goodman WK, Storch EA, et al. COVID-19-related intrusive thoughts and associated ritualistic behaviors. Rev.

41. Samuels J, HolingueC, Nestadt PS, Bienvenu OJ, Phan P, Nestadt G. Contamination-related behaviors,obsessions, and compulsions during the COVID-19 pandemic in a United Statespopulation sample. J Psychiatr Res. 2021;138:155-62.

42. Rosa-Alcázar Á, García-Hernández MD, Parada-Navas JL, Olivares-Olivares PJ, Martínez-Murillo S, Rosa-Alcázar AI. Coping strategies in obsessive-compulsive patients during Covid-19 lockdown. Int J Clin Health Psychol IJCHP. 2021;21(2):100223.

43. Pan K-Y, Kok AAL, Eikelenboom M, Horsfall M, Jörg F, Luteijn RA, et al. The mental health impact of the COVID-19 pandemic on people with and without depressive, anxiety, or obsessivecompulsive disorders: a longitudinal study of three Dutch casecontrol cohorts. Lancet Psychiatry. 2021;8(2):121-9.

44. Khosravani V, Samimi Ardestani SM, Bastan FS, McKay D, Asmundson GJG. The associations of obsessive-compulsive symptom dimensions and general severity with suicidal ideation in patients with obsessive-compulsive disorder: the role of specific stress responses to COVID-19. Clin Psychol Psychother. 2021.

45. Bahçecioğlu Turan G, Köse S, Aksoy M. Analysis of nursing students' obsessive and coping behaviors during the COVID-19 pandemic. Perspect Psychiatr Care. 2021.

46. Chen B, Sun J, FengY. How have COVID-19 isolation policies affected young people's mental health?- evidence From Chinese college students. Front Psychol. 2020;11:1529.

47. Sadri Damirchi E,Mojarrad A, Pireinaladin S, Grjibovski AM. The role of self-talk in predictingdeath anxiety, obsessive-compulsive disorder, and coping strategies in the faceof coronavirus disease (COVID-19). Iran J Psychiatry. 2020;15(3):182-8.

48. Darvishi E, GolestanS, Demehri F, Jamalnia S. A cross-sectional study on cognitive errors andobsessive-compulsive disorders among young people during the outbreak ofcoronavirus disease 2019. Act Nerv Super 2007. 2020;1-6.

49. Tandt HLN, Van ParysH, Leyman L, Purdon C, Lemmens GMD. How are OCD patients and their familiescoping with the COVID-19 pandemic? A qualitative study. Curr Psychol N B NJ.2021;1-11.

50. Capuzzi E, Di Brita C, Caldiroli A, Colmegna F, Nava R, Buoli M, et al. Psychiatric emergency care during coronavirus 2019 (COVID 19) pandemic lockdown: results from a Department of
Mental Health and Addiction of northern Italy. Psychiatry Res. 2020;293:113463.

51. El Othman R, Touma E,El Othman R, Haddad C, Hallit R, Obeid $\mathrm{S}$, et al. COVID-19 pandemic and mentalhealth in Lebanon: a cross-sectional study. Int J Psychiatry Clin Pract. 2021;1-12.

52. Wheaton MG, Prikhidko A, Messner GR. Is fear of COVID-19 contagious? The effects of emotion contagion and social media use on anxiety in response to the coronavirus pandemic. Front Psychol. 2020;11:567379.

53. Wheaton MG, Messner GR, Marks JB. Intolerance of uncertainty as a factor linking obsessive-compulsive symptoms, health anxiety and concerns about the spread of the novel coronavirus (COVID-19) in the United States. J Obsessive-Compuls Relat Disord. 2021;28:100605.

54. Seçer İ, Ulaş S. An investigation of the effect of COVID-19 on OCD in youth in the context of emotional reactivity, experiential avoidance, depression and anxiety. Int J Ment Health Addict. $2020 ; 1-14$

55. Xie M, Wang X, Zhang J, Wang Y. Alteration in the psychologic status and family environment of pregnant women before and during the COVID-19 pandemic. Int J Gynaecol Obstet Off Organ Int Fed Gynaecol Obstet. 2021;153(1):71-5.

56. McKune SL, Acosta D, Diaz N, Brittain K, Beaulieu DJ-, Maurelli AT, et al. Psychosocial health of school-aged children during the initial COVID-19 safer-at-home school mandates in Florida: a cross-sectional study. BMC Public Health. 2021;21(1):603.

57. Ahmed GK, Ramadan HK-A, Refay SM, Khashbah MA. Comparison of knowledge, attitude, socioeconomic burden, and mental health disorders of COVID-19 pandemic between general population and health care workers in Egypt. Egypt J Neurol Psychiatry Neurosurg. 2021;57(1):25.

58. Zheng Y, Xiao L, Xie Y, Wang H, Wang G. Prevalence and characteristics of obsessive-compulsive disorder among urban residents in Wuhan during the stage of regular control of coronavirus disease-19 epidemic. Front Psychiatry. 2020;11:594167.

59. Cai Z, Zheng S, Huang Y, Zhang X, Qiu Z, Huang A, et al. Emotional and cognitive responses and behavioral coping of Chinese medical workers and general population during the pandemic of COVID-19. Int J Environ Res Public Health. 2020;17(17).

60. Mrklas K, Shalaby R, Hrabok M, Gusnowski A, Vuong W, Surood S, et al. Prevalence of perceived stress, anxiety, depression, and obsessive-compulsive symptoms in health care workers and other workers in Alberta during the COVID-19 pandemic: cross-sectional survey. JMIR Ment Health. 2020;7(9):e22408.

61. Zhang W-R, Wang K, Yin L, Zhao W-F, Xue Q, Peng M, et al. Mental health and psychosocial problems of medical health workers during the COVID-19 epidemic in China. Psychother Psychosom. 2020;89(4):242-50.

62. Zheng Y, Wang L, FengL, Ye L, Zhang A, Fan R. Sleep quality and mental health of medical workersduring the coronavirus disease 2019 pandemic. Sleep Biol Rhythms. 2021;1-8.

63. Ferreira TF, Martinho F. Obsessive-compulsive disorder in times of COVID-19: a new pandemic?. Acta Med Port. 2021;34(2):167.

64. Sheu JC, McKay D, Storch EA. COVID-19 and OCD: potential impact of exposure and response prevention therapy. J Anxiety Disord. 2020;76:102314.

65. Farrell NR, Deacon BJ, Kemp JJ, Dixon LJ, Sy JT. Do negative beliefs about exposure therapy cause its suboptimal delivery? An experimental investigation. J Anxiety Disord. 2013;27(8):763-71.

66. McKay D, Minaya C, Storch EA. Conducting exposure and response prevention treatment for contamination fears during COVID-19: the behavioral immune system impact on clinician approaches to treatment. J Anxiety Disord. 2020;74:102270. 
67. Mazza MG, De Lorenzo R, Conte C, Poletti S, Vai B, Bollettini I, et al. Anxiety and depression in COVID-19 survivors: role of inflammatory and clinical predictors. Brain Behav Immun. 2020;89:594-600.

68. Mazza MG, PalladiniM, De Lorenzo R, Magnaghi C, Poletti S, Furlan R, et al. Persistentpsychopathology and neurocognitive impairment in COVID-19 survivors: effect ofinflammatory biomarkers at three-month follow-up. Brain Behav Immun. 2021;94:138-47.
69. Schou TM, Joca S, Wegener G, Bay-Richter C. Psychiatric and neuropsychiatric sequelae of COVID-19 - A systematic review. Brain Behav Immun [Internet]. 2021; Available from: https:// www.sciencedirect.com/science/article/pii/S0889159121002816.

Publisher's Note Springer Nature remains neutral with regard to jurisdictional claims in published maps and institutional affiliations. 